\title{
CONSTITUINTES QUÍMICOS E ATIVIDADE ANTIEDEMATOGÊNICA DE Peltodon radicans (LAMIACEAE)
}

\author{
Habdel Nasser Rocha da Costa \\ Departamento de Química, Centro de Ciências e Tecnologia, Universidade Federal de Roraima, Av. Capitão Ene Garcez, 2413 , \\ Campus Paricarana, 69304-000 Boa Vista - RR, Brasil \\ Maria Cristina dos Santos \\ Departamento de Parasitologia, Instituto de Ciências Biológicas, Universidade Federal do Amazonas, Campus Universitário, \\ 69077-000 Manaus - AM, Brasil \\ Antônio Flavio de Carvalho Alcântara*, Marilda Conceição Silva, Roberta Cabral França e Dorila Piló-Veloso \\ Departamento de Química, Instituto de Ciências Exatas, Universidade Federal de Minas Gerais, Av. Presidente Antônio Carlos, \\ 6627, 31260-901 Belo Horizonte - MG, Brasil
}

Recebido em 4/6/07; aceito em 11/10/07; publicado na web em 10/3/08

\begin{abstract}
CHEMICAL CONSTITUENTS AND ANTIEDEMATOGENIC ACTIVITY OF Peltodon radicans (Lamiaceae). Most of the snakebite incidents in the Amazon region involve Bothrops atrox, whose venom presents the most potent edematogenic and necrotic activities in the genus. This work describes the studies of isolation of the chemical constituents and antiedematogenic activity of the species Peltodon radicans (Lamiaceae), which is used in the treatment of snakebites and scorpion stings in the region. The extracts presented

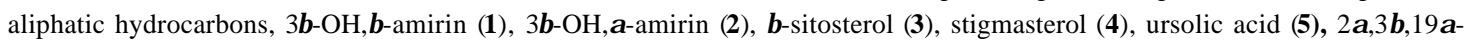
trihydroxy-urs-12-en-28-oic acid (tormentic acid, 6), methyl 3ß-hydroxy,28-methyl-ursolate (7), sitosterol-3-O- $\beta$-D-glucopyranoside (8), and stigmasterol-3-O- $\beta$-D-glucopyranoside (9). The flower extracts presented the higher antiedematogenic activity. This is the first report on the study of the flowers, stem, and roots of this plant.
\end{abstract}

Keywords: Peltodon radicans; antiedematogenic activity; triterpenes.

\section{INTRODUÇÃO}

Segundo a Organização Mundial da Saúde, anualmente cerca de 2,5 milhões de pessoas sofrem acidentes ofídicos no mundo, com 125 mil mortes. ${ }^{1}$ Esses acidentes representam um enorme problema de saúde pública, especialmente em países tropicais. ${ }^{2-4} \mathrm{Na}$ África, são registrados cerca de 500 mil acidentados por ano, com $40 \%$ dos casos hospitalizados e 20.000 óbitos. ${ }^{5} \mathrm{Na}$ Ásia há entre 25 e 35 mil óbitos por ano. ${ }^{6}$ O Ministério da Saúde do Brasil registra entre 19 e 22 mil acidentes ofídicos por ano, com aproximadamente $0,45 \%$ de letalidade. ${ }^{7}$ Quando informado o gênero da serpente, Bothrops foi responsável por 90,5\% dos acidentes ofídicos, envolvendo $0,31 \%$ de óbito.

Bothrops atrox (jararaca) é encontrada vastamente na região Norte do Brasil, ${ }^{8,9}$ habitando ambientes úmidos de zonas rurais ou periféricas das grandes cidades, onde haja facilidade para proliferação de roedores. ${ }^{10-13} \mathrm{Na}$ Amazônia essa espécie ocorre em altas densidades, principalmente em florestas, apresentando hábitos noturnos. Os jovens ingerem presas exotérmicas e os adultos, presas endotérmicas, ocorrendo portanto variação ontogenética para a peçonha dessa espécie. ${ }^{14-16}$ Os venenos de $B$. atrox são constituídos principalmente por proteínas com atividade enzimática, apresentando variações tanto inter quanto intraespecíficas. ${ }^{17-19}$ Esses vene-

Dedicamos este trabalho à Helena Ferraz, que nasceu e cresceu, e que me viu também nascer e crescer, em um daqueles muitos rincões mineiros encostados na Serra da Mantiqueira. Mantiqueira - do tupi "Amantikir" - a montanha que chora, agora chora e choramos ainda mais, por você, Helena.

*e-mail: aalcantara@zeus.qui.ufmg.br nos induzem reações locais imediatas como dor, edema, hemorragia e mionecrose,$^{20}$ podendo levar à perda tecidual abundante, assim como de parte ou de todo o membro atingido. Dentre as atividades biológicas sistêmicas destacam-se principalmente a coagulante e a hemorrágica. ${ }^{21-24}$ Os antivenenos botrópicos neutralizam principalmente os efeitos sistêmicos induzidos pelos venenos. ${ }^{25-27}$ Porém, os efeitos locais, particularmente o edema, não são neutralizados, dificultando o tratamento dos acidentados. ${ }^{28-31}$

Alguns estudos mostram o uso popular de espécies vegetais ${ }^{32-}$ ${ }^{37}$ e a eficácia da heparina no tratamento das reações locais por envenenamentos ofídicos. ${ }^{38-40}$ A espécie Peltodon radicans é conhecida popularmente por paracari, ${ }^{41-43}$ sendo encontrada na região Amazônica principalmente em terrenos arenosos e matas de terra firme. ${ }^{44,45}$ Essa espécie tem sido usada em tratamentos de asma, bronquite, coqueluche, inflamações dos rins e do fígado, afecções da pele e como vermífugo, ${ }^{46,47}$ além do uso no tratamento de acidentes ofídicos. ${ }^{48}$ Das folhas foram isolados éteres de cadeias longas, resinas, glucosídeos e alcalóides, ${ }^{49} \beta$-sitosterol, estigmasterol, ácido ursólico ${ }^{50}$ e mio-inositol ${ }^{51}$. Nenhum outro estudo fitoquímico mais sistemático sobre $P$. radicans foi encontrado na literatura. Portanto, neste trabalho são descritos os estudos do isolamento de constituintes químicos e da atividade antiedematogênica de diferentes partes dessa espécie vegetal (folhas, flores, caules e raízes) para estabelecer dentre seus princípios ativos quais atuam como antiofídicos, sendo este o primeiro relato do estudo de flores, caules e raízes desta espécie. Os estudos fitoquímicos foram direcionados para isolamento de esteróides e triterpenos pentacíclicos, considerando a vasta gama de atividades biológicas alegadas para estas duas classes de metabólitos secundários. ${ }^{52-56}$ 


\section{PARTE EXPERIMENTAL}

\section{Procedimentos fitoquímicos gerais}

Os pontos de fusão não corrigidos foram determinados em um aparelho Mettler, modelo FP80 a uma taxa de aquecimento de 2 ${ }^{\circ} \mathrm{C} / \mathrm{min}$. Os espectros de absorção na região do infravermelho (IV) foram obtidos em espectrômetro Mattson Instruments - FTIR usando pastilhas de $\mathrm{KBr}$. Os espectros de RMN foram obtidos em espectrômetro Bruker Avance DRX 400, operando na frequiência de $400 \mathrm{MHz}\left({ }^{1} \mathrm{H}\right)$ e $100 \mathrm{MHz}\left({ }^{13} \mathrm{C}\right)$, otimizados para técnicas uni (RMN de ${ }^{1} \mathrm{H}$, de ${ }^{13} \mathrm{C}$ e subespectros DEPT 135) e bidimensionais $\left({ }^{1} \mathrm{H},{ }^{1} \mathrm{H}\right.$-COSY, ${ }^{1} \mathrm{H},{ }^{1} \mathrm{H}$-NOESY, ${ }^{1} \mathrm{H},{ }^{13} \mathrm{C}$-HMQC e ${ }^{1} \mathrm{H},{ }^{13} \mathrm{C}$-HMBC). Os solventes $\mathrm{CDCl}_{3}, \mathrm{CH}_{3} \mathrm{OD}$ e $\mathrm{CD}_{5} \mathrm{~N}$ foram empregados na dissolução de quantidades variáveis de amostras, tendo TMS como padrão interno. Os deslocamentos químicos foram registrados em unidade $\delta$ e as constantes de acoplamento $(J)$ em Hz. As análises por CG/EM foram realizadas em cromatógrafo Hewlett Packard modelo HP5989A II, acoplado a espectrômetro de massas Hewlett Packard modelo 5989 A. As amostras foram energizadas a $70 \mathrm{eV}$ e submetidas à coluna metilsilicone de sílica fundida $(30 \mathrm{~m} / 0,2 \mathrm{~mm})$, tendo hélio como gás de arraste em fluxo de 1,5 mL/min.

As cromatografias de adsorção em coluna (CC) foram realizadas em colunas cilíndricas de vidro de dimensões dependentes da quantidade de amostra cromatografada, empregando como fases estacionárias sílica gel 60 (70-230 mesh) da Merck, florisil da Aldrich e Sephadex LH-20 da Sigma, submetidas a pressão atmosférica. Nas cromatografias flash foram empregadas como fases estacionárias sílica gel 60 (230-400 mesch, UETC) e florisil da Aldrich. As cromatografias em camada delgada (CCD) foram realizadas em placas de vidro recobertas por sílica gel $60 \mathrm{G}$ da Merck, empregando luz UV (254 e $366 \mathrm{~nm}$ ), vapores de iodo e solução anisaldeído/ácido sulfúrico como reveladores. Os testes de Liebermann-Burchard foram realizados para amostras dissolvidas em clorofórmio, com adição de gotas de anidrido acético e, lentamente, gotas de ácido sulfúrico concentrado, apresentando coloração violeta/azul permanente para triterpenos pentacíclicos e coloração esverdeada para esteróides. ${ }^{57}$

\section{Material vegetal}

O material vegetal de Peltodon radicans foi oriundo do município de Novo Airão, estado do Amazonas (Latitude -2,62139 e Longitude -60,94417). As coletas para estudos fitoquímicos e biológicos foram realizadas respectivamente em janeiro de 2004 e março de 2006, ambas na parte da manhã e no mesmo local, de solo argiloso e com bastante capim. Uma exsicata da espécie foi identificada pelo Dr. J. Lima (Depto. de Botânica do Instituto Nacional de Pesquisas da Amazônia - INPA), encontrando-se depositada no Herbário do INPA ( $\left.{ }^{\circ} 216.222\right)$.

\section{Extração e isolamento dos fitoconstituintes}

As diferentes partes da planta foram separadas e secadas, fornecendo materiais moídos das folhas (Fo; 613,00 g), flores $(\mathrm{Fl}$; $366,20 \mathrm{~g}$ ), caules $(\mathrm{Ca} ; 1.054,20)$ e raízes $(\mathrm{Ra} ; 206,65 \mathrm{~g})$. A extração foi realizada sucessivamente em hexano e etanol a temperatura ambiente. Após evaporação dos solventes em rotavapor, foram obtidos respectivamente os extratos hexânico $(\mathrm{EH})$ e etanólico (EE) das folhas: Fo-EH $(7,1050 \mathrm{~g})$ e Fo-EE $(54,2533 \mathrm{~g})$; flores: Fl-EH $(3,4608$ g) e Fl-EE (4,7459 g); caules: Ca-EH (37,4070 g) e Ca-EE (74,8357 g); raízes: Ra-EH (9,1710 g) e Ra-EE (2,8500 g). Os extratos foram submetidos à $\mathrm{CC}$ em coluna de sílica gel, eluindo com hexano, diclorometano, acetato de etila e etanol, em gradiente crescente de polaridade. O fracionamento cromatográfico foi acompanhado por luz UV e as frações foram reunidas em grupos de acordo com análises por CCD de sílica gel. As substâncias foram analisadas por CG/EM e RMN. A Figura 1 apresenta as estruturas químicas dos fitoconstituintes isolados de Peltodon radicans, enquanto na Tabela 1 são especificadas as suas quantidades relativas e a parte do vegetal do qual foram isolados.

As frações de Fo-EH eluídas com hexano foram reunidas e submetidas à CC em sílica gel. A eluição com hexano:diclorometano (6:4) forneceu um sólido branco $(12,8 \mathrm{mg})$, constituído por hidrocarbonetos saturados $\left(\mathrm{C}_{21} \mathrm{H}_{44}, \mathrm{C}_{12} \mathrm{H}_{26}\right.$ e $\left.\mathrm{C}_{19} \mathrm{H}_{40}\right)$. As frações hexânicas de Ca-EH forneceram um sólido branco $(65,5 \mathrm{mg})$, constituído por hidrocarbonetos saturados $\left(\mathrm{C}_{29} \mathrm{H}_{60} \mathrm{a} \mathrm{C}_{37} \mathrm{H}_{76}\right)$. As frações hexânicas de $\mathrm{Ra}-\mathrm{EH}$ foram reunidas e submetidas à $\mathrm{CC}$ em sílica gel. A eluição com hexano forneceu um sólido branco $(5,0 \mathrm{mg})$, constituído por hidrocarbonetos saturados $\left(\mathrm{C}_{27} \mathrm{H}_{56}\right.$ a $\left.\mathrm{C}_{37} \mathrm{H}_{76}\right)$.

As frações de Fl-EH eluídas com hexano foram reunidas e recristalizadas em etanol, fornecendo um sólido (11,4 mg) constituído por hidrocarbonetos saturados $\left(\mathrm{C}_{29} \mathrm{H}_{60}\right.$ a $\left.\mathrm{C}_{33} \mathrm{H}_{68}\right)$. As frações eluídas com acetato de etila foram reunidas e submetidas à $\mathrm{CC}$ de florisil. A fração eluída com diclorometano foi recristalizada em hexano:diclorometano (7:3), fornecendo um sólido $(10,1 \mathrm{mg})$ que apresentou teste Leiberman-Burchard positivo para triterpenos pentacíclicos (p.f.: $77,6-79,7^{\circ} \mathrm{C}$ ), constituído por: $3 \beta-\mathrm{OH}, \beta$-amirina $(\mathbf{1}, 42,9 \%)$ e $3 \beta-\mathrm{OH}, \alpha$-amirina $(\mathbf{2}, 57,1 \%)$.

A eluição do extrato Fo-EE com hexano:diclorometano (1:1) forneceu um sólido que foi submetido à $\mathrm{CC}$ de sílica gel. A fração eluída com hexano:diclorometano (1:1) forneceu um sólido branco $(8,6 \mathrm{mg})$, que apresentou teste Liebermann-Burchard positivo para triterpenos pentacíclicos, identificado como $\alpha$-amirina 2 . As frações de Fo-EE eluídas com diclorometano foram reunidas e submetidas à $\mathrm{CC}$ em sílica gel. A eluição com hexano:diclorometano (4:6) forneceu seis frações com sólidos brancos que foram recristalizados separadamente em etanol e apresentaram teste Liebermann-Burchard positivo para esteróides, constituídos por: Fração 1 (3 mg; 3, 69,3\%; 4, 30,7\%); Fração 2 (9,7 mg; 3, 66,1\%; 4, 33,9\%); Fração 3 (80,6 mg; 3, 65,2\%; 4, 34,8\%); Fração 4 (34,2 $\mathrm{mg} ; \mathbf{3}, 62,3 \%$; 4, 37,7\%); Fração 5 (17,7 mg; 3, 56,3\%; 4, 43,7\%); Fração 6 (140,8 mg; 3, 46,2\%; 4, 53,8\%).

As frações de Fl-EE eluídas com acetato de etila:etanol (1:1) foram reunidas e submetidas à $\mathrm{CC}$ em sílica gel. As frações eluídas com diclorometano:etanol (9:1) foram reunidas e recristalizadas em acetona, fornecendo um sólido branco $(8,5 \mathrm{mg})$, que apresentou teste Liebermann-Burchard positivo para triterpenos pentacíclicos, identificado como ácido ursólico 5. As frações eluídas com diclorometano:etanol (8:2) foram reunidas e recristalizadas em acetona, fornecendo um sólido branco (10,9 mg), que apresentou teste Liebermann-Burchard positivo para triterpenos pentacíclicos, identificado como $2 \alpha, 3 \beta, 19 \alpha$-tri-hidroxi-urs-12-en28-óico (ácido tormêntico, 6).

As frações de Ca-EE eluídas com acetato de etila foram reunidas e submetidas à $\mathrm{CC}$ de sílica gel. As frações eluídas com diclorometano foram reunidas e submetidas à $\mathrm{CC}$ de florisil. A fração eluída com hexano:diclorometano (9:1) foi recristalizada em etanol, fornecendo um sólido branco (148,5 mg), que apresentou teste Liebermann-Burchard positivo para esteróides, constituído por $3(36,5 \%)$ e 4 (63,5\%). A fração eluída com hexano: diclorometano $(8: 2)$ foi recristalizada em etanol, fornecendo um sólido branco (43,9 mg), que apresentou teste Liebermann-Burchard positivo para esteróides, constituído por $3(49,5 \%)$ e 4 (50,5\%). As frações eluídas com diclorometano:acetato de etila (1:1) foram reunidas e recristalizadas em etanol, fornecendo um sólido branco 


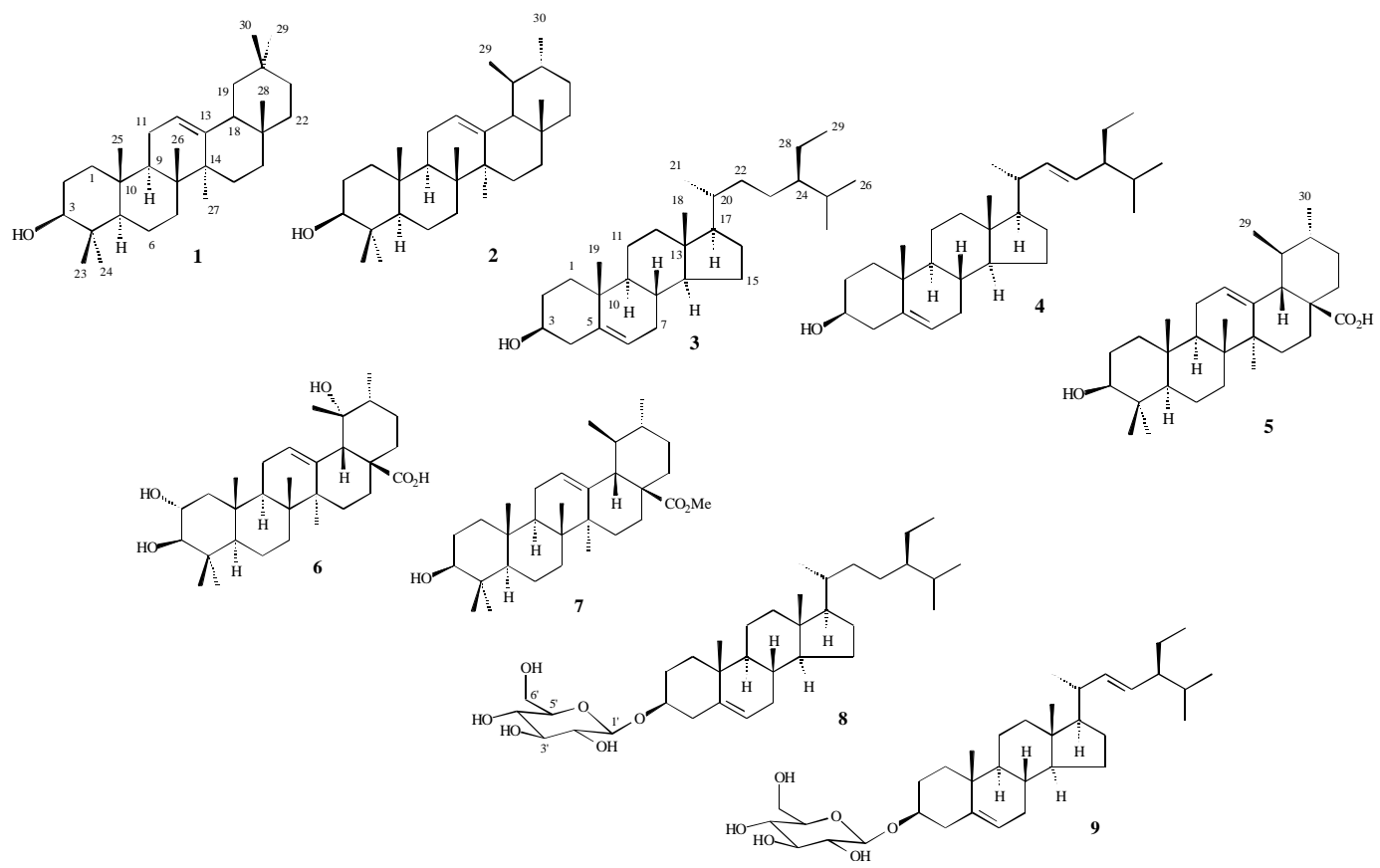

Figura 1. Estruturas químicas dos fitoconstituintes isolados de P. radicans

(45,1 mg), que apresentou teste Lieberman-Burchard positivo para triterpenos pentacíclicos, identificado como $3 \beta$-hidroxi-28-ursolato de metila (7).

As frações de Ra-EE eluídas com hexano:acetato de etila (9:1) foram reunidas e recristalizadas em metanol, fornecendo um sólido branco $(9,0 \mathrm{mg})$, que apresentou teste Liebermann-Burchard positivo para esteróides (p.f.: $146,9-148,1{ }^{\circ} \mathrm{C}$ ), constituído por 3 $(58,5 \%)$ e $4(41,5 \%)$. As frações eluídas com acetato de etila foram reunidas e recristalizadas em etanol, fornecendo um sólido branco (2,9 mg), que apresentou teste Liebermann-Burchard positivo para esteróides (p.f.: $282,9-285,6{ }^{\circ} \mathrm{C}$ ), constituído por sitosterol-3-O$\beta$-D-glicopiranosídeo $(\mathbf{8}, 31,2 \%)$ e estigmasterol-3- $O$ - $\beta$-Dglicopiranosídeo $(\mathbf{9}, 68,8 \%)$.

\section{3 $\beta$-OH, $\beta$-Amirina $(1)^{58}$}

Sólido cristalino: RMN de ${ }^{13} \mathrm{C}\left(100 \mathrm{MHz} ; \mathrm{CDCl}_{3}\right) \delta_{\mathrm{C}} 145,2$ (C-13), 121,8 (C-12), 79,1 (C-3), 55,2 (C-5), 47,8 (C-9), 47,3 (C18), 46,9 (C-19), 41,8 (C-14), 38,8 (C-1, C-4 e C-8), 37,2 (C-10 e C-22), 34,8 (C-21), 33,4 (C-29), 32,8 (C-7), 32,5 (C-17), 31,1 (C20), 28,4 (C-28), 28,1 (C-23), 27,3 (C-2), 27,0 (C-16), 26,2 (C-15), 26,0 (C-27), 23,7 (C-30), 23,6 (C-11), 18,9 (C-26), 18,4 (C-6) e 15,6 (C-24 e C-25).

\section{3 $\beta$-OH, $\alpha$-Amirina $(2)^{59}$}

Sólido cristalino: p.f.: $78,0-81,1^{\circ} \mathrm{C}$ (Lit. $\left.78,8-80,3{ }^{\circ} \mathrm{C}\right)$; RMN de ${ }^{13} \mathrm{C}\left(100 \mathrm{MHz} ; \mathrm{CDCl}_{3}\right) \delta_{\mathrm{C}} 139,6(\mathrm{C}-13), 124,4(\mathrm{C}-12), 79,1(\mathrm{C}-$ 3), 59,1 (C-18), 55,2 (C-5), 47,7 (C-9), 42,1 (C-14), 41,5 (C-22), 40,0 (C-8), 39,6 (C-19 e C-20), 39,4 (C-1), 38,8 (C-4), 36,9 (C10), 33,7 (C-17), 32,9 (C-7), 31,9 (C-21), 28,7 (C-15), 28,1 (C-23 e C-28), 27,3 (C-2), 26,6 (C-16), 23,3 (C-11), 22,7 (C-27), 21,3 (C-30), 19,8 (C-6), 17,5 (C-29), 16,9 (C-26) e 15,5 (C-24 e C-25).

\section{Ácido ursólico (5)}

Sólido cristalino: p.f.: $224,3-227,5{ }^{\circ} \mathrm{C}$ (Lit. $232,8-235,2{ }^{\circ} \mathrm{C}$ ); RMN de ${ }^{13} \mathrm{C}\left(100 \mathrm{MHz}\right.$; MeOD) $\delta_{\mathrm{C}} 181,9$ (C-28), 139,8 (C-13), 127,0 (C-12), 79,9 (C-3), 56,9 (C-5), 54,6 (C-18), 49,3 (C-17), 49,1 (C-9), 43,4 (C-14), 40,9 (C-8), 40,6 (C-4 e C-19), 40,2 (C-20), 39,9 (C-1), 38,3 (C-10 e C-22), 34,5 (C-7), 31,9 (C-21), 29,4 (C-
15), 28,9 (C-23), 28,1 (C-2), 25,5 (C-16), 24,5 (C-27), 24,2 (C-11), 21,7 (C-30), 19,4 (C-6), 17,9 (C-29), 17,7 (C-26), 16,5 (C-25) e 16,2 (C-24).

\section{Ácido $2 \alpha, 3 \beta, 19 \alpha$-tri-hidroxi-urs-12-en-28-óico (ácido tor- mêntico, 6) ${ }^{62,63}$}

Sólido branco: p.f.: $278,5-283,2{ }^{\circ} \mathrm{C}$ (Lit. $277,2-278,3{ }^{\circ} \mathrm{C}$ ); IV $\left(\mathrm{KBr}, \mathrm{cm}^{-1}\right)$ v : 3435, 2930, 2878, 1690, 1460, 1379, 1262, 1233, $1156,1048,1033,932,866$ e 802 ; RMN de ${ }^{1} \mathrm{H}$ (400 MHz; MeOD) $\delta_{\mathrm{H}} 5,20(\mathrm{~s}, \mathrm{H}-12), 3,50(\mathrm{~m}, \mathrm{H}-2), 2,81$ (d, J=9,6 Hz, H-3), 2,48 (dd, $J=13,2$ e 4,0 Hz, H-16a), 2,41 (s, H-18), 1,91 (m, H-11), 1,84 (dd, $J=12,1$ e 4,2 Hz, H-1a), 1,71 (ddd, $J=14,6,13,6$ e 4,2 Hz, H-15a), 1,65 (m, H-9), 1,63 (m, H-21a), 1,62 (m, H-22a), 1,53 (m, H-22b), 1,45 (m, H-6a), 1,42 (m, H-16b), 1,39 (m, H-6b), 1,27 (m, H-20), 1,24 (s, H-27), 1,13 (m, H-21b), 1,09 (s, H-29), 0,92 (s, H-25), 0,88 (m, H-15b), 0,84 (d, J=6,6 Hz, H-30), 0,82 (s, H-23), 0,78 (m, $\mathrm{H}-1 \mathrm{~b}), 0,77$ (m, H-5), 0,71 (s, H-24) e 0,70 (s, H-26); RMN de ${ }^{13} \mathrm{C}$ (100 MHz; MeOD) $\delta_{\mathrm{C}} 181,1$ (C-28), 140,3 (C-13), 129,4 (C-12), 84,3 (C-3), 73,8 (C-19), 69,7 (C-2), 56,9 (C-5), 55,3 (C-18), 48,8 (C-17), 48,7 (C-9), 48,3 (C-1), 43,2 (C-20), 41,3 (C-8), 40,7 (C-4), 39,4 (C-10), 39,2 (C-22), 34,3 (C-7), 29,7 (C-15), 28,8 (C-23), 27,4 (C-21), 27,2 (C-29), 26,8 (C-16), 25,0 (C-27), 24,9 (C-11), 19,8 (C-6), 17,7 (C-24), 17,6 (C-26), 17,1 (C-25) e 16,7 (C-30).

\section{3 $\beta$-Hidroxi-28-ursolato de metila $(7)^{64}$}

Sólido cristalino: p.f.: $218,9-222,7{ }^{\circ} \mathrm{C}$ (Lit. $220,0-222,0{ }^{\circ} \mathrm{C}$ ); $\mathrm{RMN}$ de ${ }^{13} \mathrm{C}\left(100 \mathrm{MHz}\right.$; MeOD) $\delta_{\mathrm{C}} 180,4$ (C-28), 138,5 (C-13), 125,3 (C-12), 78,8 (C-3), 55,3 (C-5 e O-Me), 53,0 (C-18), 47,8 (C17), 47,7 (C-9), 42,1 (C-14), 39,6 (C-8), 39,2 (C-19), 39,0 (C-20), 38,8 (C-4), 38,7 (C-1), 37,1 (C-10), 37,0 (C-22), 33,1 (C-7), 30,9 (C-21), 28,2 (C-15 e C-23), 27,3 (C-2), 24,5 (C-16), 23,6 (C-27), 23,3 (C-11), 21,3 (C-30), 18,4 (C-6), 17,1 (C-29), 17,0 (C-26), 15,7 (C-25) e 15,5 (C-24).

\section{Sitosterol-3-O $\boldsymbol{\beta} \beta$-D-glicopiranosídeo $(\mathbf{8})^{65,66}$}

Sólido cristalino: RMN de ${ }^{13} \mathrm{C}\left(100 \mathrm{MHz}\right.$; DMSO- $\left.d_{6}\right) \delta_{\mathrm{C}} 140,5$ (C-5), 121,2 (C-6), 100,8 (C-1'), 76,9 (C-3'), 76,8 (C-2'), 73,5 (C5'), 70,1 (C-3 e C-4'), 61,1 (C-6'), 56,3 (C-14), 55,3 (C-17), 49,6 
Tabela 1. Rendimento de obtenção das substâncias isoladas nos diferentes extratos de $P$. radicans

\begin{tabular}{|c|c|c|c|}
\hline $\begin{array}{l}\text { Extrato } \\
\text { (massa) }\end{array}$ & Substância & $\begin{array}{c}\text { Massa } \\
(\mathrm{mg})\end{array}$ & $\begin{array}{c}\text { Rendimento } \\
(\%)\end{array}$ \\
\hline $\begin{array}{l}\text { Fo-EH } \\
(7,1050 \mathrm{~g})\end{array}$ & Hidrocarbonetos & 12,8 & 0,18 \\
\hline Fo-EE & $\alpha$-Amirina (2) & 8,6 & 0,02 \\
\hline \multirow[t]{2}{*}{$(54,2533 \mathrm{~g})$} & $\beta$-Sitosterol (3) & 157,4 & 0,29 \\
\hline & Estigmasterol (4) & 128,6 & 0,24 \\
\hline Fl-EH & Hidrocarbonetos & 11,4 & 0,33 \\
\hline \multirow[t]{2}{*}{$(3,4608 \mathrm{~g})$} & $\beta$-Amirina (1) & 4,3 & 0,12 \\
\hline & $\alpha$-Amirina (2) & 5,8 & 0,17 \\
\hline Fl-EE & Ácido ursólico (5) & 8,5 & 0,18 \\
\hline$(4,7459 \mathrm{~g})$ & Ácido tormêntico (6) & 10,9 & 0,23 \\
\hline $\begin{array}{l}\text { Ca-EH } \\
(37,4070 \mathrm{~g})\end{array}$ & Hidrocarbonetos & 65,5 & 0,18 \\
\hline Ca-EE & $\beta$-Sitosterol (3) & 75,9 & 0,10 \\
\hline \multirow[t]{2}{*}{$(74,8357 \mathrm{~g})$} & Estigmasterol (4) & 116,5 & 0,16 \\
\hline & 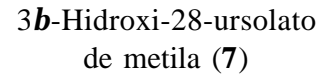 & 45,1 & 0,06 \\
\hline $\begin{array}{l}\text { Ra-EH } \\
(9,1710 \mathrm{~g})\end{array}$ & Hidrocarbonetos & 5,0 & 0,05 \\
\hline Ra-EE & $\beta$-Sitosterol (3) & 5,3 & 0,19 \\
\hline \multirow[t]{3}{*}{$(2,8500 \mathrm{~g})$} & Estigmasterol (4) & 3,7 & 0,13 \\
\hline & $\begin{array}{l}\text { Sitosterol-3-O- } \beta \text {-D- } \\
\text { glicopiranosídeo }(\mathbf{8})\end{array}$ & 0,9 & 0,03 \\
\hline & $\begin{array}{c}\text { Estigmasterol-3- } O-\beta \text {-D- } \\
\text { glicopiranosídeo }(\mathbf{9})\end{array}$ & 2,0 & 0,07 \\
\hline
\end{tabular}

$\mathrm{Fo}=$ folha; $\mathrm{Fl}=$ flor; $\mathrm{Ca}=$ caule; $\mathrm{Ra}=$ raiz; $\mathrm{EH}=$ extrato hexânico; $\mathrm{EE}=$ extrato etanólico

(C-9), 45,2 (C-24), 41,8 (C-4 e C-13), 39,2 (C-12), 36,8 (C-1), 36,2 (C-10), 35,5 (C-20), 33,4 (C-22), 31,4 (C-8), 31,3 (C-7 e C2), 28,7 (C-25), 27,8 (C-16), 25,5 (C-23), 24,9 (C-15), 22,6 (C-28), 20,6 (C-11), 19,8 (C-26), 19,1 (C-19), 18,6 (C-21 e C-27), 11,9 (C29) e $11,7(\mathrm{C}-18)$.

\section{Estigmasterol-3- $\boldsymbol{O}$ - $\boldsymbol{\beta}$-D-glicopiranosídeo $(9)^{67,68}$}

Sólido cristalino: RMN de ${ }^{13} \mathrm{C}\left(100 \mathrm{MHz}\right.$; DMSO- $\left.d_{6}\right) \delta_{\mathrm{C}} 140,5$ (C-5), 138,0 (C-22), 128,8 (C-23), 121,2 (C-6), 100,8 (C-1'), 76,9 (C-3'), 76,8 (C-2'), 73,5 (C-5'), 70,1 (C-3 e C-4'), 61,1 (C-6'), 56,2 (C-14), 55,4 (C-17), 50,6 (C-24), 49,6 (C-9), 41,9 (C-4 e C13), 40,1 (C-20), 39,2 (C-12), 36,8 (C-1), 36,2 (C-10), 31,4 (C-8 e C-25), 31,3 (C-2 e C-7), 29,3 (C-16), 24,9 (C-15 e C-28), 21,1 (C21), 20,9 (C-26), 20,6 (C-11), 19,1 (C-19), 18,6 (C-27), 12,1 (C29) e 11,8 (C-18).

\section{Atividade antiedematogênica}

Imediatamente após a coleta do material vegetal, as folhas $(317,72$ $\mathrm{g})$ e flores $(145,13 \mathrm{~g})$ foram maceradas, espremidas e os sumos liofilizados, obtendo-se os extratos macerados M-Fo (63,54 g) e MFl $(88,80$ g), respectivamente. A amostra de peçonha de Bothrops atrox foi extraída de uma fêmea adulta procedente do município de Presidente Figueiredo, estado do Amazonas. A peçonha liofilizada foi cedida pelo Núcleo de Animais Peçonhentos do Instituto de Medicina Tropical de Manaus. Os camundongos utilizados foram nãoisogênicos, fêmeas, pesando entre 18 e $22 \mathrm{~g}$, procedentes do Biotério do Departamento de Ciências Fisiológicas - ICB, UFAM. Esses animais foram mantidos em gaiolas de plástico a temperatura ambiente, recebendo ração apropriada e água à vontade.
Para determinação da atividade bloqueadora da ação edematogênica do veneno de Bothrops atrox ${ }^{69}$ foram formados sete grupos com quatro animais por grupo. Seis grupos de camundongos receberam os extratos de M-Fo ou M-Fl nas doses de 7,0, 3,5 e $1,75 \mathrm{mg} / \mathrm{mL}$, e um grupo foi usado como controle. As suspensõesmãe utilizadas nos preparos das doses foram feitas pesando-se de cada extrato $70 \mathrm{mg}$, que foram diluídos, separadamente, em $1 \mathrm{~mL}$ de solução salina. As doses dos extratos testadas nos experimentos corresponderam às usadas popularmente nos acidentes por serpentes peçonhentas em humanos. Todos os grupos de camundongos receberam a dose equivalente a três vezes a dose mínima edematogênica (DME) do veneno $(3 \times 1,2 \mu \mathrm{g} /$ animal - anteriormente determinada para esta amostra de veneno), no coxim plantar da pata traseira direita (pata experimental), em um volume de 50 $\mu \mathrm{L}$. No coxim plantar, da pata contralateral (pata controle) foram aplicados $50 \mu \mathrm{L}$ de solução salina. As diferentes doses dos extratos vegetais foram injetadas por via intraperitoneal no tempo zero, em um volume de $500,0 \mu \mathrm{L}$. Os animais do grupo controle receberam solução salina $(500,0 \mu \mathrm{L})$ pela via intraperitoneal. As mensurações das patas foram realizadas nos tempos de 2, 4 e 6 h após aplicação do veneno, com auxílio do paquímetro digital Mitutoyo. O edema foi expresso em percentagem, pela diferença entre as espessuras do coxim da pata experimental e da pata controle, dividida pela espessura do coxim da pata controle, multiplicado por 100, conforme Equação 1 (onde $\mathrm{V}_{\mathrm{p}} \%$ é o aumento percentual do volume podal, sendo $\mathrm{V}_{\mathrm{f}}$ e $\mathrm{V}_{\mathrm{i}}$ os volumes final e inicial das patas medidas, respectivamente)

$\mathrm{V}_{\mathrm{p}} \%=\left[\left(\mathrm{V}_{\mathrm{f}}-\mathrm{V}_{\mathrm{i}}\right) / \mathrm{V}_{\mathrm{i}}\right] \times 100$

Alíquotas dos extratos obtidos da primeira coleta foram submetidas aos testes de bloqueio da ação edematogênica do veneno de Bothrops atrox. Os extratos Fo-EE e Ca-EE $(100 \mu \mathrm{L})$ foram diluídos em $900 \mu \mathrm{L}$ de solução salina. O extrato Fl-EE $(100 \mu \mathrm{L})$ foi diluído em $800 \mu \mathrm{L}$ de solução salina e em $100 \mu \mathrm{L}$ de Twenn 20. Os experimentos seguiram a mesma metodologia descrita acima.

\section{RESULTADOS E DISCUSSÃO}

\section{Estudo fitoquímico}

Todos os hidrocarbonetos saturados isolados foram caracterizados por CG/EM. O esqueleto de triterpenos pentacíclicos do tipo oleonano de $\mathbf{1}$ foi proposto pela análise dos dados de RMN e comparação com dados da literatura para $3 \beta-\mathrm{OH}-\beta$-amirina ${ }^{58} \mathrm{O}$ carbono metílico C-28 de 2 foi confirmado pela correlação HMBC do sinal em $\delta_{\mathrm{H}} 5,13$ (m, H-18) com o sinal de carbono em $\delta_{\mathrm{C}} 28,1$ (C-28). Análises por RMN indicaram o esqueleto de triterpenos pentacíclicos do tipo ursano para 2, estando de acordo com dados descritos na literatura para $\alpha$-amirina. ${ }^{59}$ As estruturas dos esteróides $\mathbf{3}$ e $\mathbf{4}$ foram determinadas pelas análises por RMN e comparação com dados descritos na literatura para $\beta$-sitosterol ${ }^{70}$ e estigmasterol, ${ }^{71}$ respectivamente. Análises por RMN indicaram o esqueleto de triterpenos pentacíclicos do tipo ursano para $\mathbf{5}$, estando de acordo com dados descritos na literatura para ácido ursólico. ${ }^{60,61}$

A caracterização estrutural de $\mathbf{6}$ foi baseada em análises por RMN 1D e 2D. Pelo mapa de contornos HMBC verificou-se que H-12 $\left(\delta_{\mathrm{H}} 5,20\right)$ apresentou correlações com os carbonos de $\delta_{\mathrm{C}} 140,3$ (C-13), 55,3 (C-18), 48,7 (C-9), 43,0 (C-14) e 24,9 (C-11) e pelo mapa de contornos COSY com os hidrogênios de $\delta_{\mathrm{H}} 2,41$ (s, H-18) e 1,91 (m, H-11). O hidrogênio H-18 $\left(\delta_{\mathrm{H}} 2,41\right)$ correlaciona-se por HMBC com os carbonos de $\delta_{\mathrm{C}} 181,9$ (C-28), 140,3 (C-13), 129,4 (C-12), 73,8 (C-19), 43,2 (C-20), 43,0 (C-14), 39,2 (C-22), 27,2 
(C-29) e 26,8 (C-16), enquanto por NOESY apresenta correlações com os hidrogênios de $\delta_{\mathrm{H}} 0,70$ (s, H-26) e 1,09 (s, H-29). Esse último hidrogênio correlaciona-se por $\mathrm{HMBC}$ com os carbonos de $\delta_{\mathrm{C}} 73,8(\mathrm{C}-19), 55,3$ (C-18) e 43,2 (C-20) e ainda por NOESY apresenta correlações com o hidrogênio de $\delta_{\mathrm{H}}$ 1,27-1,24 (m, H-20).

$\mathrm{O}$ hidrogênio $\mathrm{H}-2\left(\delta_{\mathrm{H}} 3,50\right)$ correlaciona-se por HMBC com os carbonos de $\delta_{\mathrm{C}} 84,3$ (C-3), 48,3 (C-1), 40,7 (C-4) e 39,4 (C-10), apresentando por COSY correlações com os hidrogênios de $\delta_{\mathrm{H}} 2,81$ (d, $J=9,6 \mathrm{~Hz}, \mathrm{H}-3$ ), 1,84 (dd, $J=12,1$ e 4,2 Hz, H-1a) e 0,78 (m, H1b) e por NOESY correlações com os hidrogênios de $\delta_{\mathrm{H}} 0,82(\mathrm{~s}, \mathrm{H}-$ 23), 0,92 (s, H-25) e 0,78 (m, H-1b).

O hidrogênio H-3 $\left(\delta_{\mathrm{H}} 2,81\right)$ correlaciona-se por HMBC com os carbonos de $\delta_{\mathrm{C}} 69,7$ (C-2), 56,9 (C-5), 48,3 (C-1), 40,7 (C-4), 28,8 (C-23) e 17,7 (C-24), apresentando por NOESY correlações com os hidrogênios de $\delta_{\mathrm{H}} 0,77$ (m, H-5) e 1,84 (dd, $J=12,1$ e 4,2 Hz, H-1a). O hidrogênio $\mathrm{H}-25\left(\delta_{\mathrm{H}} 0,92\right)$ apresenta por HMBC correlações com os carbonos de $\delta_{\mathrm{C}} 56,9$ (C-5), 48,3 (C-1), 39,4 (C-10) e 48,7 (C-9), apresentando por NOESY correlação com o hidrogênio de $\delta_{H} 0,70(\mathrm{~s}, \mathrm{H}-$ 26). Esse último hidrogênio apresenta por HMBC correlações com os carbonos de $\delta_{\mathrm{C}} 48,7$ (C-9), 43,0 (C-14), 41,3 (C-8) e 34,3 (C-7). As demais atribuições dos sinais de $\mathrm{RMN}$ de ${ }^{1} \mathrm{H}$ e de ${ }^{13} \mathrm{C}$ do ácido tormêntico foram realizadas por comparação com dados da literatura. $^{62,63}$

Análises por RMN indicaram também o esqueleto de triterpenos pentacíclicos do tipo ursano para 7 , estando de acordo com dados descritos na literatura para $3 \beta$-hidroxi-28-ursolato de metila ${ }^{64}$. As estruturas dos esteróides 8 e $\mathbf{9}$ foram determinadas pelas análises por RMN e comparação com dados descritos na literatura para sitosterol-3-O- $\beta$-D-glicopiranosídeo ${ }^{65,66}$ e estigmasterol-3- $O-\beta$-Dglicopiranosídeo, ${ }^{67,68}$ respectivamente.

A Tabela 1 mostra o rendimento de obtenção das substâncias isoladas nos diferentes extratos da planta. Misturas dos esteróides 3 e 4 foram isoladas de Fo-EE, Ca-EE e Ra-EE, sendo também isolados os esteróides glicosilados 8 e 9 neste último extrato. Porém, esses esteróides não foram encontrados nas flores. Triterpenos pentacíclicos foram isolados em maiores proporções nas flores, sendo 1 e $\mathbf{2}$ de Fl-EH e $\mathbf{5}$ e $\mathbf{6}$ de Fl-EE. Proporções, relativamente, pequenas dos triterpenos pentacíclicos $\mathbf{2}$ e $\mathbf{7}$ foram isolados respectivamente de Fo-EE e Ca-EE.

\section{Atividade antiedematogênica}

A Tabela 2 mostra os resultados obtidos nos testes de bloqueio da ação edematogênica com os extratos Fo-EE, Fl-EE e Ca-EE e as mensurações das patas em diferentes tempos após as aplicações. Após 2 h, apenas Fl-EE apresentou ação bloqueadora, pois reduziu 23,39\% da atividade edematogênica do veneno de Bothrops atrox, em relação aos animais controle. Essa ação bloqueadora foi mais eficaz na $4^{\mathrm{a}}$ e $6^{\mathrm{a}} \mathrm{h}(34,67$ e 57,38\%, respectivamente). Já o extrato Ca-EE apresentou fraca ação bloqueadora na dose testada $\left(2^{\mathrm{a}} \mathrm{h}=\right.$ $17,33 \% ; 4^{\mathrm{a}} \mathrm{h}=7,44 \%$ e na $6^{\mathrm{a}} \mathrm{h}=20,89 \%$ ) e o extrato Fo-EE não foi eficaz em bloquear a ação edematogênica induzida pelo veneno de B. atrox. Os valores negativos do bloqueio da ação edematogênica apresentados nesta tabela podem ser interpretados como potencialização do efeito edematogênico.

A Tabela 3 expressa os resultados obtidos dos testes de bloqueio da ação edematogênica 2 e 4 h após a aplicação dos extratos obtidos de acordo com o uso popular de M-Fo e M-Fl nas concentrações de 7,0; 3,5 e 1,75 mg/mL em solução salina. Apenas na $4^{\text {a }}$ h, M-Fo apresentou atividade bloqueadora nas concentrações de 1,75 e 7,0 mg/mL. Esses resultados indicam efeito pouco eficaz de M-Fo na neutralização da atividade edematogênica do veneno.

Por outro lado, M-Fl apresentou atividade antiedematogênica
Tabela 2. Bloqueio da atividade edematogênica do veneno de Bothrops atrox pelos extratos Fl-EE, Fo-EE e Ca-EE em relação aos animais controle, nos diferentes tempos após a aplicação

\begin{tabular}{lccc}
\hline Tempo após aplicação & \multicolumn{3}{c}{$\%$ de Bloqueio dos Extratos } \\
& Fo-EE & Fl-EE & Ca-EE \\
\hline $2 \mathrm{~h}$ & $-49,65$ & 23,39 & $-17,33$ \\
$4 \mathrm{~h}$ & $-51,45$ & 34,67 & 7,44 \\
$6 \mathrm{~h}$ & $-24,62$ & 57,38 & 20,89 \\
\hline
\end{tabular}

$\mathrm{Fo}=$ folha; $\mathrm{Fl}=$ flor; $\mathrm{Ca}=$ caule; $\mathrm{Ra}=$ raiz; $\mathrm{EH}=$ extrato hexânico; $\mathrm{EE}=$ extrato etanólico.

efetiva, dose-dependente, nos diferentes tempos observados. Portanto, os testes indicam que as flores apresentam maior concentração de composto(s) eficaz(es) em bloquear a ação edematogênica do veneno de Bothrops atrox que os extratos obtidos das outras partes da espécie vegetal (folhas e caule).

Tabela 3. Bloqueio da atividade edematogênica do veneno de Bothrops atrox pelos extratos M-Fo e M-Fl em relação aos animais controle, em concentrações variáveis e diferentes tempos após a aplicação

\begin{tabular}{|c|c|c|c|}
\hline \multirow{2}{*}{$\begin{array}{l}\text { Tempo após } \\
\text { aplicação }\end{array}$} & \multirow{2}{*}{$\begin{array}{l}\text { Concentração } \\
(\mathrm{mg} / \mathrm{mL})\end{array}$} & \multicolumn{2}{|c|}{$\%$ de Bloqueio dos Extratos } \\
\hline & & M-Fo & $\mathrm{M}-\mathrm{Fl}$ \\
\hline \multirow[t]{3}{*}{$2 \mathrm{~h}$} & 1,75 & 1,05 & 49,74 \\
\hline & 3,5 & 1,22 & 60,36 \\
\hline & 7,0 & 1,66 & 65,19 \\
\hline \multirow[t]{3}{*}{$4 \mathrm{~h}$} & 1,75 & 15,16 & 57,26 \\
\hline & 3,5 & 26,97 & 61,90 \\
\hline & 7,0 & 14,57 & 82,73 \\
\hline
\end{tabular}

$\mathrm{M}-\mathrm{Fo}=$ macerado das folhas; $\mathrm{M}-\mathrm{Fl}=$ macerado das flores.

Estudos mostram que esteróides apresentam atividades antiinflamatórias, ${ }^{72}$ podendo ser relacionadas com a neutralização do efeito letal dos venenos brotópicos. ${ }^{73}$ No entanto, os extratos das flores não forneceram os esteróides $3, \mathbf{4 ,} 8$ e 9 (Tabela 1) mas apresentaram maiores efeitos de DME (Tabelas 2 e 3). Assim sendo, não é possível relacionar essas substâncias com a atividade DME estudada das flores.

Os efeitos anti-hiperalgésico de $\mathbf{2}$ têm sido observados nas dores inflamatórias persistentes. ${ }^{74}$ Alguns estudos mostram que 1 e 2 apresentam atividades similares aos corticóides. ${ }^{75}$ A atividade antiinflamatória dos triterpenos tem sido atribuída à presença de grupos carboxílicos e hidroxílicos em posições específicas nas interações de receptores glucocorticóides. Nos casos de $\mathbf{1}$ e $\mathbf{2}$, a atividade antiinflamatória tem sido atribuída à posição do grupo hidroxila em C-3, com efeitos antiinflamatórios localizados. ${ }^{76}$ Nos extratos das flores foram encontrados esses dois triterpenos (Tabela 1), o que poderia ser relacionado com a atividade antiedematogênica de Fl-EE e M-Fl (Tabelas 2 e 3).

$\mathrm{O}$ ácido ursólico 5 é encontrado amplamente no reino vegetal, apresentando diversas propriedades farmacológicas, como antiedematogênica e antiinflamatória. ${ }^{77-82}$ Por sua vez, o ácido tormêntico 6 é menos freqüente em espécies vegetais, ${ }^{83-86}$ apresentando também atividade antiinflamatória. ${ }^{87}$ Esses dois triterpenos foram encontrados apenas nas flores a 0,18 e $0,23 \%$, respectivamente. Ambas as proporções são relativamente altas, permitindo relacionar 5 e $\mathbf{6}$ com a atividade antiedematogênica de Peltodon radicans verificada popularmente e confirmada pelos testes biológicos realizados neste trabalho. 


\section{CONCLUSÃO}

Nos extratos das folhas, caules e raízes de Peltodon radicans foram identificados os esteróides $\mathbf{3}, \mathbf{4}, \mathbf{8}$ e 9, além de proporções relativamente pequenas dos triterpenos pentacíclicos $\mathbf{2}$ e 7. Esses extratos apresentaram menores atividades antiedematogênicas que o extrato das flores. Os testes de DME indicaram que o extrato das flores de Peltodon radicans apresentou eficácia significativa na neutralização da atividade edematogênica do veneno de Bothrops atrox. Além disso, a ação bloqueadora da atividade edematogênica desse extrato aumentou tanto com o tempo de aplicação quanto com a concentração do extrato (dose-resposta). Portanto, os resultados estão de acordo com algumas das informações etnofarmacológicas dessa espécie vegetal. Os triterpenos pentacíclicos 1, 2, 5 e 6, identificados nos extratos das flores, podem estar relacionados com o bloqueio da atividade edematogênica pela $P$. radicans.

\section{AGRADECIMENTOS}

Ao Conselho Nacional de Desenvolvimento Científico e Tecnológico (CNPq), à Fundação de Amparo à Pesquisa do Estado de Minas Gerais (FAPEMIG) e à Fundação de Amparo à Pesquisa do Estado do Amazonas (FAPEAM) pelo suporte financeiro.

\section{REFERÊNCIAS}

1. Cardoso, J. C. L.; Brando, R. B.; Acidentes por animais peçonhentos. Ed Santos: São Paulo, 1982.

2. Pinho, F. M. O.; Oliveira, E. S.; Faleiros, F.; Rev. Assoc. Med. Bras. 2004, $50,16$.

3. Ministério da Saúde do Brasil; Manual de Diagnóstico e Tratamento de Acidentes por Animais Peçonhentos, Fundação Nacional de Saúde: Brasília, 1998.

4. Pinho, F. M. O.; Pereira, I. D.; Rev. Ass. Bras. 2001, 47, 24.

5. Chippaux, J. P.; Bull. World Health Org. 1998, 76, 515.

6. Ribeiro, L. A.; Albuquerque, M. U.; Campos, V. A. P.; Rev. Saúde Pública 1995, 5, 380 .

7. Ministério da Saúde do Brasil; Manual de Diagnóstico e Tratamento de Acidentes por Animais Peçonhentos, Fundação Nacional de Saúde: Brasília, 2001.

8. Jorge, M. T.; Ribeiro, L. A.; Rev. Inst. Med. Trop. 1992, 34, 347.

9. Campbell, J. A.; Lamar, W. W.; The Venomous Reptiles of Latin America, Cornell University: New York, 1989.

10. Barraviera, B.; Arq. Bras. Med. 1991, 65, 345.

11. Hoge, A. R.; Hoge, S. A. R. W. R.; Mem. Inst. Butantan 1978/79, 42/43, 373.

12. Cunha, R.; Ofídios da Amazônia, Museu Emílio Goeldi: Belém, 1978.

13. Dos-Santos, M. C.; Martins, M.; Boechat, A. L.; Sá-Neto, R. P.; Oliveira, M. E.; Serpentes de interesse médico da Amazônia, UFAM/SESU: Manaus, 1995.

14. Cardoso, J. L.; França, F. O. S.; Wen, F. H.; Sant'Ana, C. M. M.; Haddad, Jr. V.; Animais Peçonhentos no Brasil: Biologia, clínica e terapêutica dos acidentes, Sarvier: São Paulo, 2003.

15. http://eco.ib.usp.br/labvert/Jararaca/projjar_atrox_manaus.htm, acessada em Março 2007.

16. Bolaños, R.; Serpientes, Venenos y Ofidismo en Centro América, Ed. Univ. de Costa Rica: San José, 1984.

17. Furtado, M. F. D.; Colleto, G. M. D. D.; Dias da Silva, W.; Mem. Inst. Butantan 1991, 53, 149.

18. Meier, J.; Toxicon 1996, 34, 787.

19. Otero, R.; Osorio, R. G.; Valderrama, R.; Giraldo, O. A.; Toxicon 1992 30,611 .

20. Russel, F. E.; Calson, R. W.; Wainschel, J.; Osborne, A. H.; JAMA, J. Am. Med. Assoc. 1975, 233, 341.

21. Rosenfeld, G. Em Venomous Animals and their Venoms; Buchel, W.; Buckley, E., eds.; Academic Press: New York, 1971.

22. Teixeira,C. F. P.; Cury, Y.; Olga, S.; Jancar, S.; Toxicon 1994, 32, 419.

23. Cury, Y.; Teixeira, C. F. P.; Sudo, L.; Toxicon 1994, 32, 1425.

24. Picolo, G.; Chacur, M.; Gutiérrez, J. M.; Teixeira, C. F. P.; Cury, Y.; Braz. J. Med. Biol. Res. 2002, 35, 1221.

25. Warrell, D. A.; The Global Problem of Snake Bite: Its Prevention and Treatment, Nat. Univ. of Singapore: Singapura, 1992.
26. Coetzer, P. W.; Tilbury, C. R.; S. Afr. Med. 1982, 62, 206.

27. Cardoso, J. L. C.; Fan, H. W.; Franca, F. O. S.; Jorge, M. T.; Leite, R. P.; Nishioka, S. A.; Avila, A.; Sano-Martins, I. S.; Tomy, S. C.; Santoro, M. L.; Chudzinski, A. M.; Castro, S. B.; Kamiguti, A. S.; Kelen, E. M. A.; Hirata, M. H.; Mirandola, R. M. S.; Theakston, R. D. G.; Warrell, D. A.; Q. J. Med. 1993, 86, 315.

28. Gutiérrez, J. M.; Chaves, F.; Bolaños, R.; Cerdas, L.; Rojas, E.; Arroyo, O.; Portilla, E.; Toxicon 1981, 19, 493.

29. Gutiérrez, J. M.; Gené, J. A.; Cerdas, L.; Rojas, G.; Toxicon 1985, 23, 887.

30. Gutiérrez, J. M.; Gené, J. A.; Lemonte, B.; Cerdas, L.; Rojas, G.; Comp. Biochem. Physiol., Part C: Toxicol. Pharmacol. 1986, 85, 171.

31. Jorge, M. T.; Ribeiro, L. A.; Rev. Ass. Med. Brasil. 1997, 43, 74.

32. Melo, P. A.; Nascimento, M. C.; Mors, W. B.; Suarez-Kurtz, G.; Toxicon 1994, 32, 595.

33. Mors, W. B.; Nascimento, M. C.; Pereira, B. M. R.; Pereira, M. A.; Phytochemistry 2000, 55, 627.

34. Freitas, F. G.; Silva, T. A.; Oliveira, F.; Santos, B. R.; Homsi-Brandeburgo, M. I.; Hamaguchi, A.; Biosci. J. 2005, 21, 95.

35. Veronese, E. L.; Esmeraldino, L. E.; Trombone, A. P.; Santana, A. E.; Bechara, G. H.; Kettlelhut, I.; Cintra, A. C.; Giglio, J. R.; Sampaio, S. V.; Phytomedicine 2005, 12, 123.

36. Neves, P. C. A.; Maria Neves, M. C.; Cruz, A. B.; Sant'ana, A. E. G.; Yunes, R. A.; Calixto, J. B.; Eur. J. Pharmacol. 1993, 243, 213.

37. Borges, M. H.; Alves, D. L.; Raslan, D. S.; Piló-Veloso, D.; Rodrigues, V. M.; Homsi-Brandenburgo, M. I.; Lima, E.; J. Ethnopharmacol. 2005, 98 , 21.

38. Borges, C. C.; Sadahiro, M.; Dos-Santos, M. C.; Rev. Soc. Bras. Med. Trop. 1999, 32, 637 .

39. Melo, P. A.; Suarez-Kurtz, G.; Toxicon 1988, 26, 87.

40. Melo, P. A.; Homsi-Brandeburgo, M. I.; Giglio, J. R.; Suarez-Kurtz, G.; Toxicon 1993, 31, 285.

41. Lorenzi, H.; Matos F. J. A.; Plantas Medicinais no Brasil, Nativas e Exóticas, Nova Odessa: Instituto Plantarum, Rio de Janeiro, 2002.

42. http://www.zum.de/stueber/pohl/band1/high/IMG_6485.html, acessado em Março 2007.

43. Balbach, A.; A Flora Nacional na Medicina Doméstica, Edel - A Edificação do Lar: São Paulo, 1969.

44. Pedersen, J. A; Biochem. Syst. Ecol. 2000, 28, 229.

45. Riberiro, J. E. L. S.; Hopkins, M. J. G.; Vicentini, A.; Sothers, C. A.; Costa, M. A. S.; Brito, J. M.; Souza, M. A. D.; Martins, L. H. P.; Lohmann, L. G.; Assunção, P. A. C. L.; Pereira, E. C.; Silva, C. F.; Mesquita, M. R.; Procópio, L. C.; Flora da Reserva Ducke: Guia de Identificação das Plantas Vasculares de uma Floresta de Terra Firme na Amazônica Central, INPA: Manaus, 1999.

46. Barberán, F. A. T.; Barkmeijer, R. J. G.; Gil, M. I.; Harbonrne, J. B.; Phytochemistry 1988, 27, 2631.

47. Paiva, E. A. S.; Machado, S. R.; Braz. Arch. Biol. Techn. 2005, 48, 147.

48. Ruppelt, B. M.; Pereira, E. F.; Gonçalves, L. C.; Pereira, N. A.; Mem. Inst. Oswaldo Cruz 1991, 86, 203.

49. Peckolt, T.; J. Chem. Soc. 1905, 11, 113.

50. Zelnik, R.; Matilda, K. A.; Paniza, S.; Mem. Inst. Butantan 1978/1979, 42/ 43, 357.

51. Moniz, A. M. H.; Dissertação de Mestrado, Universidade Federal do Amazonas, Brasil, 2004.

52. Oliveira, B. H.; Bueno, D. D.; Quim. Nova 1996, 19, 233.

53. Patoèka, J.; J. Applied Biom. 2003, 1, 7.

54. Singh, B.; Singh, S.; Phytother. Res. 2003, 17, 814

55. Cárdenas, C.; Quesada, A. R.; Medina, M. A.; Biochem. Biophy. Res. Commun. 2004, 320, 402.

56. Liu, J.; J. Ethnopharmacol. 1995, 49, 57.

57. Matos, F. J. A.; Introdução à Fitoquímica Experimental, Edições UFC: Fortaleza, 1997.

58. Lima, F. V.; Malheiros, A.; Otuki, M. F.; Calixto, J. B.; Yunes, R. A.; Filho, V. C.; Monache, B. F. D.; J. Braz. Chem. Soc. 2005, 16, 578.

59. Otuki, F. M.; Ferreira, J.; Lima, F. V.; Meyre-Silva, C.; Malheiros, A.; Muller, L. A.; Cani, G. S.; Santos, A. R. S.; Yunes, R. A.; Calixto, J. B.; J. Pharmacol. Exp. Ther. 2004, 10, 1124.

60. Mahato, S. B.; Kundu, A. P.; Phytochemistry 1994, 37, 1517.

61. Romeo, G.; Gianetto, P.; Aversa, M. C.; Org. Magn. Reson. 1977, 9, 29

62. Taniguchia, S.; Imayoshia, Y.; Kobayashia, E.; Takamatsua, Y.; Itoa, H.; Hatanoa, T.; Sakagamib, H.; Tokudac, H.; Nishinoc, H.; Sugitad, D.; Shimurad, S.; Yoshida, T.; Phytochemistry 2002, 59, 315.

63. Tanaka, J. C. A.; Vidotti, G. J.; Silva, C. C.; J. Braz. Chem. Soc. 2003, 14, 475.

64. Seo, B. S.; Tomita, Y.; Tori, K.; J. Chem. Soc., Chem. Commun. 1975, 954.

65. Kojima, H.; Sato, N.; Hatano, A.; Ogura, H.; Phytochemistry 1990, 29, 2351. 
66. Silva, D. A.; Silva, T. M. S.; Lins, A. C. S.; Costa, D. A.; Cavalcante, J. M. S.; Matias, W. N.; Souza, M. F. V.; Braz-Filho, R.; Quim. Nova 2006, 29, 1250.

67. Paula, V. F.; Barbosa, L. C. A.; Demuner, A. J.; Piló-Veloso, D.; Howarth, O. W.; Eclética Química 1998, 23, 45.

68. Agrawal, P. K.; Phytochemistry 1982, 31, 3307.

69. Yamakawa, M.; Nozaki, M.; Hakana, Z.; Plants Microbial Toxins 1976, 1, 98.

70. Garg, V. K.; Nes, W. R.; Phytochemistry 1984, 23, 2925.

71. Goulart, M. O. F.; Sant'Ana, A. E. G.; Lima, R. A.; Cavalcante, S. H.; Carvalho, M. G.; Braz-Filho, R.; Quim. Nova 1993, 16, 95.

72. Mbaze, L. M.; Poumale, H. M. P.; Wansi, J. D.; Lado, J. A.; Khan, S. N.; Iqbal, M. C.; Ngadjui, B. T.; Laatsch, H.; Phytochemistry 2007, 68, 591.

73. Mors, W. B.; Nascimento, M. C.; Pereira, B. M. R.; Pereira, M. A.; Phytochemistry 2000, 55, 627.

74. Hasmeda, M.; Kweifio-Okai, G.; Macrides, T.; Polya, G.; Planta Med. 1999, 65, 14 .

75. Tolstikov, G. A.; Baltina, L. A.; Serdyuk, N. G.; Pharm. Chem. J. 1998, 32,5 .

76. Recio, M.; Giner, R.; Máñez, S.; Gueho, J.; Julien, H.; Hostettmann, K.; Ríos, J.; Planta Med. 1995, 61, 9.
77. Ovesna, Z.; Vachalkova, A.; Horvathova, K.; Tothova, D.; Neoplasma 2004, 51,327 .

78. Miceli, N.; Taviano, M. F.; Giuffrida, D.; Trovato, A.; Tzakou, O.; Galati, E. M.; J. Ethnopharmacol. 2005, 97, 261.

79. Ngouela, S.; Ndjakou, B. L.; Tchamo, D. N.; Zelefack, F.; Tsamo, E.; Connolly, J. D.; Nat. Prod. Res. 2005, 19, 23.

80. Jeong, H. G.; Kim, H. G.; Hwang, Y. P.; Toxicol. Lett. 2005, 155, 369.

81. Ryu, S. Y.; Oak, M. H.; Yoon, S. K.; Cho, D. I.; Yoo, G. S.; Kim, K. M.; Planta Med. 2000, 66, 358.

82. Banno, N.; Akihisa, T.; Tokuda, H.; Yasukawa, K.; Higashihara, H.; Ukiya, M.; Watanabe, K.; Kimura, Y.; Hasegawa, J.; Nishino, H.; Biosci., Biotechnol., Biochem. 2004, 68, 85.

83. Beirith, A.; Santos, A. R. S.; Calixto, J. B.; Hess, S. C.; Messana, I.; Ferrari, F.; Yunes, R. A.; Planta Med. 1999, 65, 50.

84. Hess, S. C.; Monache, F. D.; J. Braz. Chem. Soc. 1999, 10, 104.

85. Houghton, P. J.; Lian, L. M.; Phytochemistry 1986, 25, 1939.

86. Lee, K. H.; Lin, Y. M.; Wu, T. S.; Zhang, D. C.; Yamagishi, T.; Hayashi, T.; Hall, I. H.; Chang, J. J.; Wu, R.Y.; Yang, T. H.; Planta Med. 1988, 54, 308 .

87. Bortalanza, L. B.; Ferreira, J.; Hess, C. S.; Monache, D. F.; Yunes, A. R.; Calixto, J. B.; Eur. J. Pharmacol. 2002, 453, 203. 